\title{
Agricultural Management Affects Root-Associated Microbiome Recruitment Over Maize Development
}

\author{
Cassandra J. Wattenburger, ${ }^{1}$ Larry J. Halverson, ${ }^{1, \dagger}$ and Kirsten S. Hofmockel ${ }^{2}$ \\ ${ }^{1}$ Department of Plant Pathology and Microbiology, lowa State University, Ames, IA 50011 \\ ${ }^{2}$ Department of Ecology, Evolution, and Organismal Biology, lowa State University, Ames, IA 50011 and Environmental Molecular Sciences \\ Laboratory, Pacific Northwest National Laboratory, Richland, IA 99354
}

Accepted for publication 30 July 2019.

\section{ABSTRACT}

Diversified cropping systems provide yield benefits that may result from enhanced nutrient availability via the root microbiome. We hypothesized that root-associated microbial communities in diversified and conventional systems would differ most during high plant nitrogen demand, reflecting microbiome-derived benefits, and that these effects would be greatest nearest the root. We compared maize bulk soil, rhizosphere, and rhizoplane prokaryotic and fungal communities in diversified and conventional systems at four plant developmental stages using amplicon sequencing. The greatest differences between systems in root-selected prokaryotic community composition occurred within the rhizoplane during vegetative stage 11 (high nitrogen demand). During this period, the rhizoplane of maize from the diversified, compared with the conventional, cropping system hosted higher abundances of bacteria implicated in complex organic matter decomposition
(Verrucomicrobia and Acidobacteria). In contrast, fungal rootassociated communities differed most between cropping systems during vegetative stage 4 (low nitrogen demand) and became more similar over time. Unexpectedly, prokaryotic rhizosphere communities, unlike rhizoplane communities, did not always differentiate from bulk soil communities, indicating value in differentiating between root compartments to understand root-microbiome-management interactions. For example, the diversified system rhizosphere and bulk soil prokaryotic communities were not well differentiated compared with the conventional system, indicating the possibility of a camouflage effect.

Keywords: management, microbiome, nutrient cycling, rhizosphere and phyllosphere, soil ecology
Nutrient use inefficiency is a pressing problem in modern agriculture. An estimated 198.1 million tons of NPK fertilizers will be applied to agricultural soils around the world in 2019, with increasing global consumption projected for coming years (FAO

Current address for first author: Department of Soil and Crop Sciences, Cornell University, Ithaca 14853

\section{${ }^{\dagger}$ Corresponding author: L. Halverson; larryh@isatate.edu}

Funding: This research was supported by the National Institute of Food and Agriculture, USDA AFRI grant program (grant number 2014-67019-2168) and by the Iowa Agriculture and Home Economics Experiment Station. This material is based upon work supported by Larry Halverson while serving at the U.S. National Science Foundation (NSF). Any opinion, findings, and conclusions or recommendations expressed in this material are those of the author(s) and do not necessarily reflect the views of the U.S. NSF.

*The $e$-Xtra logo stands for "electronic extra" and indicates that 11 supplementary figures, three supplementary tables, and three supplementary files are published online.

The author(s) declare no conflict of interest.

(C) 2019 The American Phytopathological Society
2017). Despite the large quantity of fertilizers being applied, only a fraction may be assimilated by crops: as little as $50 \%$ of nitrogen (N) and 10\% of phosphorus (Baligar et al. 2001; Meena et al. 2017). Among these nutrients, $\mathrm{N}$ is the most extensively applied globally (Smil 1999). Its mismanagement has resulted in serious adverse side effects such as nitrous oxide efflux and nitrate leaching, which contribute to the greenhouse effect and fresh and saltwater pollution, respectively (Alexander et al. 2008; Carpenter et al. 1998; Niu et al. 2016; Vitousek et al. 1997). The environmental impact of our fertilization practices may be reduced with management practices that better couple fertilizer application to crop nutrient uptake.

Ecologically minded management may hold potential to address nutrient use inefficiency. Plants in unfertilized terrestrial systems mainly acquire $\mathrm{N}$ and other nutrients via the depolymerization of soil organic matter by microorganisms (Schimel and Bennett 2004). A mutualistic interaction between plant roots and microbes exists in which plants exude carbon (C), in the form of organic acids, peptides, lipids, and carbohydrates, which stimulates the activity of microorganisms near the root, increasing nutrient turnover from organic matter (Berendsen et al. 2012; Kraiser et al. 2011; Richardson et al. 2009). As plants develop, the root-associated 
microbiome shifts as well, likely due to changes in root $\mathrm{C}$ exudation (Baudoin et al. 2002; Chiarini et al. 1998; Gomes et al. 2003; Hannula et al. 2012; Houlden et al. 2008; Micallef et al. 2009; Pfeiffer et al. 2017; Wieland et al. 2001). The Arabidopsis thaliana rhizosphere microbiome's $\mathrm{N}$ metabolism gene expression was shown to change with shifts in root $\mathrm{C}$ exudation (Chaparro et al. 2014). This suggests that interactions between plant rhizodeposition and the root microbiome are one mechanism by which plants may meet nutritional needs as they grow. Despite this naturally occurring process, conventional agroecosystems mainly rely on external inputs of inorganic nutrients to create a bioavailable supply to crops. As such, there is interest in harnessing root-associated microbial communities to enhance nutrient use efficiency in agricultural systems (Adesemoye and Kloepper 2009), but there is still work to be done to understand how plant nutrient demand and rootassociated microbiomes interact as crops grow.

In order to effectively manage rhizosphere microbial communities for plant nutrition, we must understand how agricultural management affects the selection of root-associated communities. It has been demonstrated that agricultural management effects crop rhizosphere microbial communities (Fernandez et al. 2016a, b; Gdanetz and Trail 2017; Hargreaves et al. 2015; Hartman et al. 2018). A pot and field experiment showed that management of inorganic $\mathrm{N}$ inputs influenced patterns of maize rhizodeposition, and these changes corresponded to shifts in the rhizosphere microbial community composition (Zhu et al. 2016). Together, these studies show that careful consideration of soil management is likely necessary to improve nutrient management in agroecosystems via the microbiome. However, more detailed investigations of how soil management, crop nutrient demand, and rhizosphere communities interact will be necessary to make rhizosphere microbiome-mediated nutrient use efficiency a reality.

We also lack detailed studies of the rhizosphere microbiome that differentiates between differing compartments of the rhizosphere. Root exudates appear to play an important role in shaping the rhizosphere microbiome (Chaparro et al. 2013, 2014; Micallef et al. 2009). Root exudates may diffuse up to $5 \mathrm{~mm}$ from the root surface but are also thought to be rapidly consumed by soil microbiota or otherwise adsorbed to the soil matrix (Beck and Gilmour 1983; Kuzyakov et al. 2003; Watt et al. 2006). Rhizoplane communities, those on the root surface, are likely exposed to higher concentrations of exudates compared with less closely associated rhizosphere communities due to higher concentrations of root exudates within the first $2 \mathrm{~mm}$ of the root (Kuzyakov et al. 2003), which may shape their structures or functions. The most closely associated root microorganisms may be important to plant microbiome-mediated nutrient uptake considering the advantage that microorganisms have over plants for recently mobilized N (Kuzyakov and Xu 2013). Despite this, most investigations of rhizosphere communities do not make distinctions between differing compartments such as the endosphere, rhizoplane, and the less closely associated rhizosphere communities.

Lastly, agricultural diversification is one means by which the interactions between plant root selection and microbial activities may be managed to improve nutrient use efficiency, but few experiments have explicitly investigated the connections between management, rhizosphere community selection, and crop nutrient demand. These interactions may be studied at experimental field sites such as the Marsden Long-Term Cropping System Experiment located in Boone County, IA. Despite large differences in fertilization practices, the diversified cropping system (4-year rotation with reduced tillage, pesticides, and inorganic fertilization) at Marsden is just as productive as the conventional cropping system (2-year rotation with exclusively inorganic fertilization) (Davis et al. 2012). Prior work showed that the diversified system at the Marsden site supports higher levels of microbial biomass, greater production of bioavailable $\mathrm{N}$ from SOM, potentially mineralizable $\mathrm{N}$, and reduced nitrate pool sizes in a depth-dependent manner (King and Hofmockel 2017; Osterholz et al. 2018, 2017; Tomer and Liebman 2014). Gross ammonification rates in bulk soil did not differ substantially between the conventional and diversified systems, leading the authors to conclude that soil $\mathrm{N}$ availability does not play a major role in boosting yields in the diversified systems but that plant-microbiome interactions may play an unspecified role (Osterholz et al. 2018). These documented ecosystem services allow us to use the Marsden site as a model for exploring how agricultural management affects the coupling between microbial communities and plant roots, and how this interaction may factor into increased nitrogen use efficiency in agriculture.

In this work, we explored three main questions relating to the impacts of agricultural management on root microbiome selection: (i) do management and plant nutrient demand interact to impact root selection of the microbiome; (ii) if so, how far-reaching are these effects from the root; and (iii) which management-specific differences in the root-associated microbiomes may be relevant to the nutrient uptake of the plant? We hypothesized that differences in the root-associated microbial communities between the Marsden diversified and conventional agricultural systems (Davis et al. 2012) would be greatest during high nutrient (N) demand because, if coupling between roots and microbes occurs based on nutrient demand, the community best-suited to meeting nutritional needs would be selected based on the types of nutrients available and the microbial seedbank as shaped by agricultural management. We also hypothesized that these differences would be strongest at the rhizoplane due to a greater relative concentration of $\mathrm{C}$ exudates that stimulate microbial growth. To test these hypotheses, we assessed the prokaryotic and fungal communities of the maize rhizoplane (directly adhered microorganisms and soil particles), rhizosphere soil (soil particles clinging to the root), and bulk soil (no living root influence) within conventional and diversified systems at Marsden. We sampled at various maize developmental stages corresponding to high or low relative $\mathrm{N}$ uptake, using $\mathrm{N}$ demand to guide our sampling due to the relative importance of $\mathrm{N}$ as a limiting nutrient and its outsized environmental impacts.

\section{MATERIALS AND METHODS}

Field site description. This study was conducted at the Iowa State University Marsden Long-Term Cropping System Experiment located in Boone County, IA $\left(42^{\circ} 01^{\prime} \mathrm{N} ; 93^{\circ} 47^{\prime} \mathrm{W} ; 333 \mathrm{~m}\right.$ above sea level) (Davis et al. 2012). The Marsden site was established in 2002, and prior to that, was used for conventional corn-soybean agriculture. The soil at the site is mostly comprised of Clarion loam, Nicollet loam, and Webster silty clay loam (Davis et al. 2012; Supplementary Fig. S1). We sampled from two management regimes: conventional and diversified. The conventional treatment, managed with a 2-year crop rotation of maize (Zea mays) and soybean (Glycine max) with inorganic $\mathrm{N}$ fertilization, is similar to surrounding Iowan farms. The diversified treatment instead employs a 4-year rotation of maize, soybean, oat (Avena sativa)/alfalfa (Medicago sativa), and alfalfa and receives most of its fertilization from composted manure and, when warranted, a reduced inorganic $\mathrm{N}$ fertilizer side dress. The conventional system was fertilized with $100 \mathrm{lbs}$ of UAN 32 per acre 1 day prior to planting (05/05/2016), while the diversified system was fertilized with 7.6 tons of composted manure per acre the previous fall. 
Thirty-seven days after planting, a side dress of 90 and $60 \mathrm{lbs}$ of UAN 32 was applied to the conventional and diversified systems, respectively. Tillage and pesticide management also differ between systems, with reduced frequency and intensity of each in the diversified system (Davis et al. 2012; Liebman et al. 2008). The Marsden site is a completely randomized block design comprised of $83.8 \mathrm{~m}$ by $18.3 \mathrm{~m}$ plots and for this study, we sampled blocks 1 to 3 .

Sampling protocol. We sampled during plant growth stages with contrasting predicted $\mathrm{N}$ demand: vegetative stages 4 and 11 (V4 and $\mathrm{V} 11$ ), which corresponded to low and high $\mathrm{N}$ demand, and reproductive stages 2 and 5 (R2 and R5), which corresponded to high, then low relative predicted $\mathrm{N}$ demand (Licht et al. 2011). After planting (05/06/2016), we randomly chose three sampling spots in each plot, and all subsequent samples were taken from within a $10-\mathrm{ft}$ radius. Due to high spatial heterogeneity of soil samples, samples taken from different sampling spots within the same block were kept separate and treated independently, with blocking effects taken into account for statistical analysis. This resulted in $n=9$ samples per treatment at each time point (three samples taken per block) for a total of 216 samples by the end of the experiment. Sampling occurred on 9 June, 29 June, 26 July, and 1 September 2016, corresponding to $390,901,1,519$, and 2,331 growing degree days. At the time of sampling, corn plants were staged using the Leaf Collar method to verify developmental stage (Licht et al. 2011). If heavy precipitation occurred, we delayed sampling for at least 1 week to reduce the effects of soil moisture on the microbiome.

We collected bulk and rhizosphere soil, and rhizoplane samples using the following protocols. Three bulk soil cores of the top $15 \mathrm{~cm}$ of soil were taken from each sampling spot halfway between rows directly adjacent to the plant used for rhizosphere and rhizoplane sampling. Bulk soil cores from the same sampling spot were homogenized and subsampled into 2-ml centrifuge tubes and Whirl-Pak bags for DNA extraction and soil property analysis, respectively (Supplementary File S3; Supplementary Tables S1 and S2). Samples for DNA extraction were frozen immediately on dry ice in the field prior to long-term storage at $-80^{\circ} \mathrm{C}$.

We used a shovel to uproot the plant in the top layer $(15$ to $20 \mathrm{~cm})$ of soil and shook the root ball several times to remove large, loosely adhered soil aggregates. We collected rhizosphere soil from roots via shaking and gentle scraping. Tweezers were used to remove small roots and other visible debris from the rhizosphere soil, which was then transferred into 2-ml centrifuge tubes and immediately frozen on dry ice prior to storage at $-80^{\circ} \mathrm{C}$. At R2 and R5 sampling times, a large root-ball had developed that would not loosen with shaking. In this case, rhizosphere soil was taken from accessible roots extending from this root ball. After collection of rhizosphere soil, roots were placed in Whirl-Pak bags, frozen on dry ice, and stored at $-80^{\circ} \mathrm{C}$.

The rhizoplane was defined as the fine soil particles and microbes that adhered to the root after rhizosphere soil collection. For stages V4 and V11, the rhizoplane was sampled from the whole root, and for stages R2 and R5, the rhizoplane was sampled from 5 to $10 \mathrm{~cm}$ long randomly selected healthy root segments equivalent in biomass to root samples collected from stage V11. Frozen root samples were placed in 40 to $45 \mathrm{ml}$ of an ice-cold phosphate buffer solution $(0.1 \mathrm{M})$, vortexed for $30 \mathrm{~s}$ and then decanted over a $100 \mu \mathrm{m}$ nylon mesh filter into an empty 50-ml falcon tube. Another $45 \mathrm{ml}$ of icecold phosphate buffer solution was added to the decanted falcon tube containing the roots and then sonicated for $30 \mathrm{~s}$ at $20 \mathrm{~W}$ using a needle probe to remove attached soil and cells. This sonicated buffer solution was poured over another $100 \mu \mathrm{m}$ nylon mesh and the filtrates from both steps were pooled. The pooled filtrate was centrifuged at 10,000 RPM for 5 min at $4{ }^{\circ} \mathrm{C}$ and then decanted, leaving a pellet of soil and root-adherent microbes that was then transferred to microcentrifuge tubes and frozen at $-20^{\circ} \mathrm{C}$ before being stored at $-80^{\circ} \mathrm{C}$.

Amplicon sequencing. DNA was extracted from $0.25 \mathrm{~g}$ of fresh weight of sample material using MoBio PowerLyzer PowerSoil kits (Mobio Laboratories, Carlsbad, CA). DNA samples were sent to the Next Generation Sequencing Core at Argonne National Laboratory (Lemont, IL) for amplicon library preparation and sequencing using the $16 \mathrm{~S}$ V4 region targeted by the $515 \mathrm{~F} / 806 \mathrm{R}$ primer set and the fungal internal transcribed spacer (ITS) region 1 using a modified ITS1F (5' CTTGGTCATTTAGAGGAAGTAA 3') and ITS2 primer set (5' GCTGCGTTCTTCATCGATGC 3') (Smith and Peay 2014). Amplicons of $16 S$ and ITS were paired-end sequenced (read lengths of $150 \mathrm{bp} \times 150 \mathrm{bp}$ and $250 \mathrm{bp} \times 250 \mathrm{bp}$, respectively) on an Illumina MiSeq platform on separate runs. Mock community samples were also included to evaluate sequencing biases and verify taxonomic resolution. Three replicates of the ZymoBIOMICS Mock Community DNA Standard (Zymo Research, Irvine, CA) or three replicates of fungal mock community DNA (Bakker 2018) were included with the $16 \mathrm{~S}$ and ITS libraries.

Data analysis. Sequences, processed using the open source pipeline hundo (Brown et al. 2018), were quality filtered using BBDuk2 to remove adapter sequences and PhiX at a matching kmer length of $31 \mathrm{bp}$ and hamming distance of 1 (Bushnell 2014). At this time, all reads shorter than $51 \mathrm{bp}$ were discarded. USEARCH was used to merge reads with a minimum length of $175 \mathrm{bp}$ and maximum error rate of $1 \%$ (Edgar 2010). Sequences were dereplicated and clustered using USEARCH at $97 \%$ sequence identity (distancebased, greedy clustering method) (Edgar 2013). Chimeric sequences were predicted de novo at the same time. Taxonomic assignment was performed using BLAST alignments and then assigned by least common ancestor across the SILVA database version 123 for $16 \mathrm{~S}$ sequences or UNITE version 7 for ITS sequences (Camacho et al. 2009; Kõljalg et al. 2013; Quast et al. 2013). We used USEARCH on seed sequences filtered against SILVA version 123 or UNITE version 7 for $16 \mathrm{~S}$ and ITS, respectively, to find chimeric operational taxonomic units (OTUs). Raw sequences were deposited to NCBI short read archives (accession SRP129432).

Singleton OTUs were discarded and counts were cumulative sum scaling normalized to the 75 th percentile to mitigate sequencing depth bias (Paulson et al. 2013). Three fungal samples were discarded as failed sequencing efforts because they fell below a previously defined threshold of 10 OTU richness and 1,000 OTU counts prior to normalization. Unknown phyla, chloroplast, and mitochondrial OTUs were removed prior to downstream analyses.

Statistical analyses were performed in $\mathrm{R}$ version 3.3 using the packages 'vegan', 'labdsv', 'phyloseq', and 'DESeq2' (Anders and Huber 2010; Love et al. 2014; McMurdie and Holmes 2013; Oksanen et al. 2017; R Core Team 2017; Roberts 2016). All scripts are available at https://github.com/cwatt/DEV-project-R-code. Treatment effects on richness, defined as the total number of OTUs present in the sample, and Shannon's diversity indices were tested via Kruskal-Wallis tests. Distance matrices were created at the OTU-level using Bray-Curtis dissimilarity, and then nonmetric multidimensional scaling (NMDS) ordinations were created from those distance matrices. Treatment effects on $\beta$-diversity were determined using permutational analysis of variance (PERMANOVA) with permutations constrained within each block. Multiple comparisons using PERMANOVA and a Bonferroni $P$-adjustment were used to determine differences in community composition between contrasts of interest. 
We used the package 'DESeq2' to identify differentially abundant taxa between treatments (Anders and Huber 2010; Love et al. 2014). We did analyses on both the OTU and family-level count data. To account for multiple comparisons, DESeq2 applies the Benjamin-Hochberg adjustment to limit the false positive discovery rate. Additionally, we denoted significance at an adjusted $P$ value of 0.01 or less. When comparing rhizoplane or rhizosphere soil communities between cropping systems, we filtered out differentially abundant taxa that were not also differentially abundant in the rhizosphere or rhizoplane compared with the corresponding bulk soil. This limited our findings to taxa that were differentially abundant within the rhizosphere soil and rhizoplane due to the influence of the root and not simply due to differences in the bulk soil environment.

Mock communities were analyzed by averaging the triplicate counts for each OTU then iteratively searching for each community member at each taxonomic level (Supplementary Table S3). For each community member, we calculated the ratio of that member relative to the whole community and compared that to the expected ratio as defined in the mock community. This provided a means for assessing sequencing biases and the accuracy of taxonomic assignment. Within the prokaryotic mock community, only Lactobacillus fermentum was severely under-represented and taxonomic assignment was successful primarily at the genus or family level (seven out of eight), with one exception at the order level. Only 10 of 18 fungal mock community species were identified correctly at the family level and three Fusarium species were undifferentiated from one another.

\section{RESULTS}

Major prokaryotic and fungal phyla. Illumina MiSeq sequencing resulted in 3,635,209 ITS and 8,664,921 16S reads with an average of 16,908 $\pm 5,968$ (SD) ITS and 38,856 $\pm 10,441$ (SD) $16 \mathrm{~S}$ reads per sample. There were a total of 19,842 and 3,136 unique OTUs identified from $16 \mathrm{~S}$ and ITS sequencing respectively. In both the conventional and diversified systems, the most relatively abundant prokaryotic phyla, on average, were the Proteobacteria, Acidobacteria, Bacteroidetes, and Verrucomicrobia but with differing proportions based on cropping system, proximity to the root, and plant developmental stage (Fig. 1A). The most relatively abundant fungal phyla were Ascomycota, Zygomycota, and Basidiomycota, of which various fungal classes varied by cropping system, developmental stage, and root proximity (Fig. 1B).

Prokaryotic and fungal diversity. Both species richness and Shannon's diversity indices of the prokaryotic community differed significantly as the plant developed $(P<0.05)$ but did not differ by cropping system or root proximity (Fig. 2A). Over the course of the growing season, prokaryotic $\alpha$-diversity decreased overall. Fungal Shannon's diversity, but not richness, significantly differed by plant developmental stage $(P<0.0001)$, but not other variables, and Shannon's diversity decreased over the growing season (Fig. 2B; Supplementary Fig. S2). In all cases, there was a block effect $(P<$ $0.05)$, possibly reflecting the heterogeneity of soil series at this site.

Cropping system, development, and root proximity effects on microbial community composition. Cropping system, root proximity, and plant developmental stage influenced prokaryotic and fungal community composition at the OTU level, with some significant interactions (Table 1). Prokaryotic and fungal communities separate clearly by proximity to the root (horizontal spread) and less clearly by cropping system (vertical spread) and plant developmental stage (Fig. 3). Rhizoplane communities exhibited greater shifts in composition across maize developmental stages compared with communities at other root proximity levels
(Fig. 4). Due to the relatively strong effect of the root proximity by developmental stage interaction, we further explored differences in community composition at each root-proximity level and developmental stage by conducting specific contrasts (Tables 2 and 3 ). These analyses revealed that root-associated microbial communities varied over plant developmental stage and cropping system uniquely.

Differential abundance of specific microbial taxa relative to root proximity. We performed differential abundance analyses at the OTU and family levels to further evaluate how microbial community composition differed between cropping systems at each root proximity level. The bulk soils of the two cropping systems had the greatest number of differentially abundant prokaryotic OTUs at the R2 stage, while differentially abundant taxa between rhizosphere soils or rhizoplanes peaked at stage V11 (Fig. 5). In contrast, fungal communities had the greatest number of differentially abundant taxa at stage V4 and decreased thereafter (Fig. 5). Next, we further highlight differences in microbial communities between cropping systems at each root-proximity level.

Bulk soil. The bulk soils of each cropping system harbored distinct prokaryotic and fungal communities (Table 2; Fig. 3) that did not differ significantly between consecutive plant developmental stages (Table 3). At the OTU level at each sampling time there were over 100 differentially abundant prokaryotic taxa between bulk soil communities (Supplementary Fig. S3; Supplementary File S1). The phyla with the greatest number of OTUs that were differentially abundant between the cropping systems were the Proteobacteria, Bacteroidetes, Actinobacteria, and Acidobacteria. While some differentially abundant taxa stayed consistently enriched or suppressed throughout the growing season, others were differentially abundant only once or twice. For example, three Thaumarchaeota OTUs were differentially abundant between cropping systems at all developmental stages and were mostly more abundant within the conventional system except for one OTU at later stages of plant growth. Planctomycetes, Verrucomicrobia, and Acidobacteria OTUs tended to be more abundant in the diversified cropping system bulk soil. Other bacterial phyla did not follow clear trends of enrichment between cropping systems, such as the Proteobacteria, Bacteroidetes, and Gemmatimonadetes, which had many OTU representatives enriched in both systems.

At each developmental stage, there were over 45 differentially abundant fungal OTUs between the bulk soils of the two cropping systems, with 10 different Ascomycota classes comprising most of the differentially abundant taxa (Supplementary Fig. S4). Ascomycota had many OTUs enriched in either system, while other taxa, such as within the Zygomycota, were commonly more abundant in the diversified system (specifically the family Mortierellaceae).

Rhizosphere. The rhizosphere soil of each cropping system harbored distinct fungal and prokaryotic communities at each stage of plant development (Table 2; Fig. 3). While the prokaryotic rhizosphere communities in either cropping system did not vary significantly between consecutive plant developmental stages, the fungal rhizosphere communities did so in a cropping system specific manner (Table 3; Fig. 4). Fungal rhizosphere and bulk soil communities did not differ significantly in the conventional system at V4 and V11 but did at R2 and R5 (Table 2). In the diversified system, fungal rhizosphere community composition only differed significantly from the bulk soil at R5 (Table 2). The diversified system prokaryotic rhizosphere soil communities were not statistically different from the bulk soil communities at any plant developmental stages after adjusting for multiple comparisons (Table 2). This contrasts with the conventional system, where the bulk and rhizosphere soil prokaryotic community structures were significantly different at stages V11 and R2 (Table 2). This is also 
evident in the greater number of differentially abundant OTUs between bulk and rhizosphere soils in the conventional compared with the diversified system at these developmental stages (Fig. 6; Supplementary File S2).

In both the conventional and diversified system rhizosphere soils, there was an overall enrichment for Proteobacteria, Verrucomicrobia, and Bacteroidetes OTUs compared with that in the bulk soils (Fig. 6). At every developmental stage, there was a greater number of differentially abundant OTUs between the rhizosphere soil community and bulk soil community in the conventional system compared with in the diversified system. Notably, of the differentially abundant Proteobacteria OTUs in the conventional system rhizosphere compared with that in the bulk soil, $35 \%$ were $\alpha$-Proteobacteria. In comparison, only $12 \%$ of the differentially abundant OTUs in the diversified system rhizosphere soil compared with in the bulk soil were $\alpha$-Proteobacteria. All but one enriched Burkholderiales ( $\beta$-Proteobacteria) OTUs and all enriched Pseudomonadaceae ( $\gamma$-Proteobacteria) OTUs in the diversified rhizosphere soil compared with in the bulk soil were also enriched in the conventional system, but there were also Burkholderiales and Pseudomonadaceae OTUs enriched in the conventional system rhizosphere soil compared with in the bulk soil that were not also enriched in the diversified system.
Differences between bulk soil and rhizosphere soil fungal communities were due to shifts in the abundances of only a few (1 to 15) OTUs in each system and at each time point, mainly due to OTUs within the Ascomycota (Supplementary Fig. S7). Rhizosphere taxa that differed in abundance between cropping systems included prokaryotic representatives within the Proteobacteria, Verrucomicrobia, Acidobacteria, Actinobacteria, and Bacteroidetes (Supplementary Fig. S5) and fungal representatives of the Ascomycota, Zygomycota, and Basidiomycota (Supplementary Fig. S6).

Rhizoplane. The rhizoplanes of each cropping system harbored distinct fungal and prokaryotic communities that varied significantly between consecutive plant developmental stages, with a few exceptions for the fungal community (Table 3; Fig. 4). In both cropping systems, prokaryotic and fungal rhizoplane communities differed significantly from both the bulk and rhizosphere soil communities at all plant developmental stages, with an exception between the fungal rhizosphere and rhizoplane communities at stage R2 (Table 2; Fig. 3). Fungal rhizoplane communities differed significantly between systems at stage V4 and R5 (Table 2). However, rhizoplane prokaryotic communities followed a different pattern in which composition significantly differed between systems at V4 and V11 but did not differ during R2 and R5 stages (Table 2).
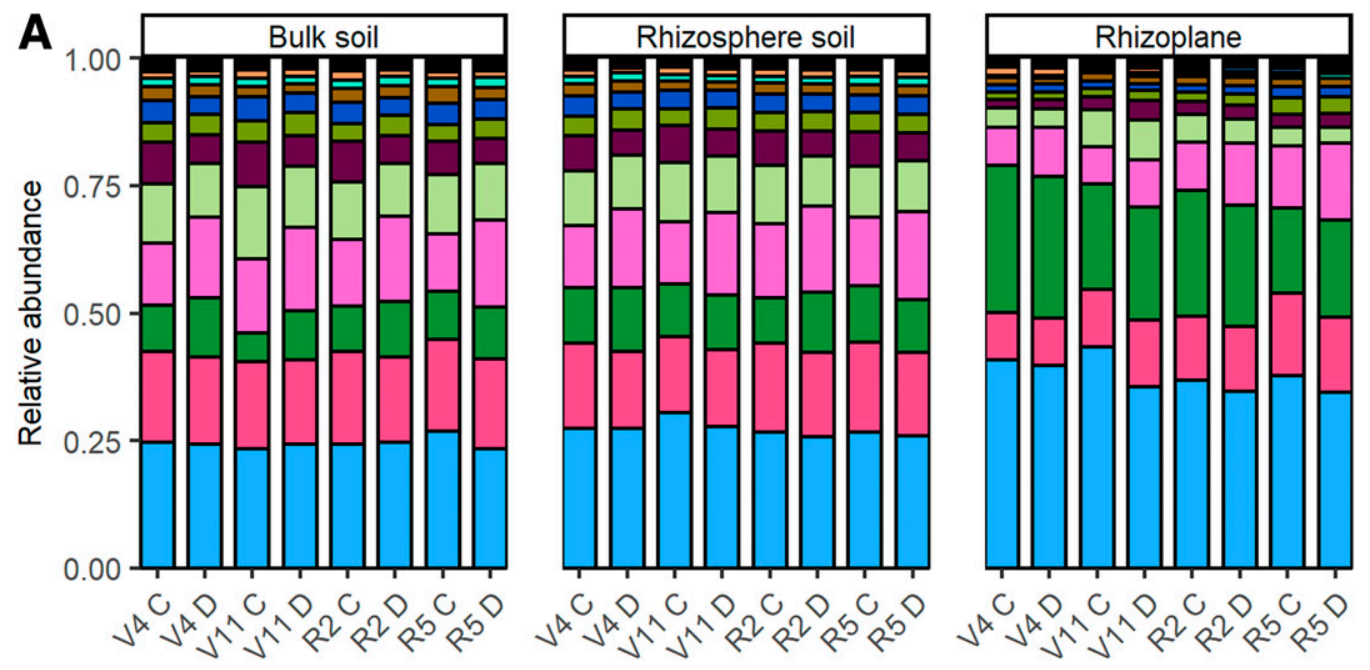

\section{Phylum}

Proteobacteria
Acidobacteria
Bacteroidetes
Verrucomicrobia
Actinobacteria
Thaumarchaeota
Planctomycetes
Chloroflexi
Gemmatimonadetes
Nitrospirae
Firmicutes
Armatimonadetes
Latescibacteria
Elusimicrobia
Chlorobi
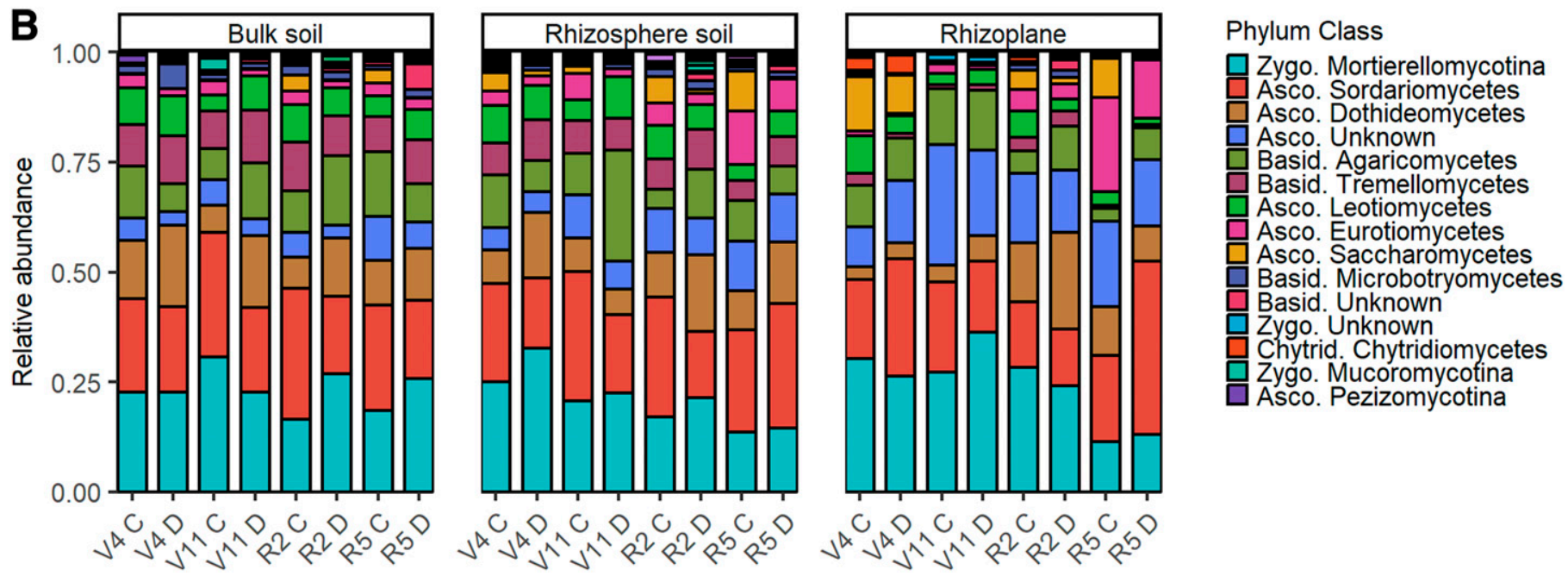

Fig. 1. Relative abundance of A, prokaryotic and B, fungal communities across plant developmental stage, cropping system, and proximity to root. The legends list only the top 15 most abundant taxa. Fungal phyla are abbreviated as Asco. (Ascomycota), Basid. (Basidiomycota), Zygo. (Zygomycota), and Chytrid. (Chytridomycota). C and D in the x-axis labels refer to conventional and diversified cropping system, respectively. 
There were intriguing differences in the number and distribution of differentially abundant OTUs in the rhizoplane compared with in the bulk soils of each cropping system (Supplementary Fig. S8). There were 25 prokaryotic phyla with differentially abundant OTUs with some, such as the Thaumarchaeota, Nitrospira, and Gemmatimonadetes, usually more abundant in the bulk soil compared to in the rhizoplane. Approximately 47 to $48 \%$ of the differentially abundant OTUs between the rhizoplane and bulk soil communities were members of the Proteobacteria and Bacteroidetes. Notably, there was a substantially greater number of differentially abundant OTUs between rhizoplane and bulk soil in the conventional system at the V4 and V11 stages in comparison with in the diversified system at those stages.

Between rhizoplane prokaryotic communities in the two systems, the largest number of differentially abundant OTUs between systems occurred at the V11 stage (Supplementary Fig. S11). Of the differentially abundant Bacteroidetes OTUs, 60 to $82 \%$ were members of the family Chitinophagaceae while the rest represented by three to five other families. There was strong selection for various Proteobacteria in the conventional rhizoplane. Moreover,
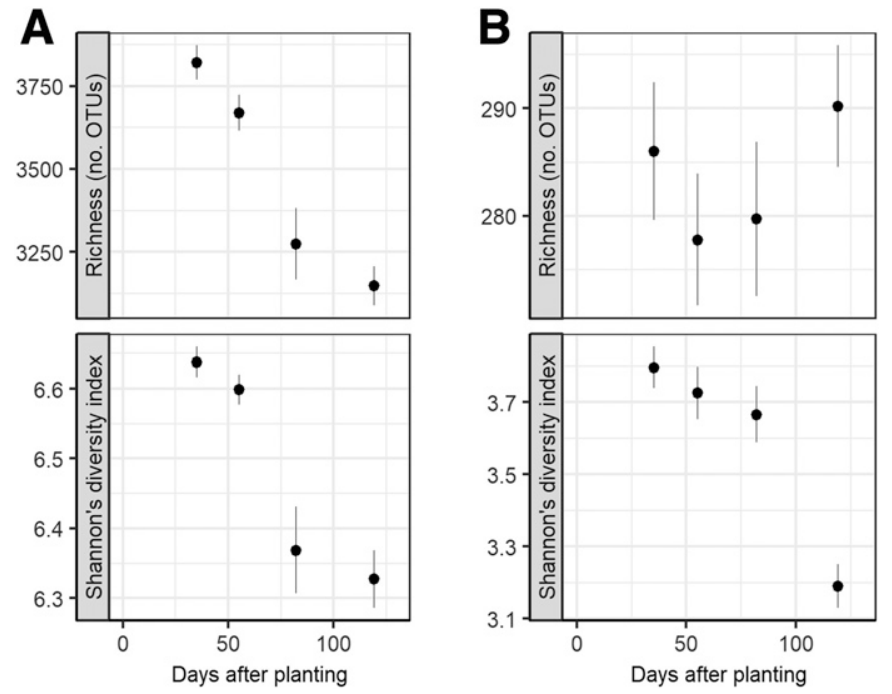

Fig. 2. Richness and Shannon's diversity indices over time. A, Prokaryotic and $\mathbf{B}$, fungal communities averaged over cropping system and root-proximity level. Points indicate means and bars indicate standard error. The main effect of developmental stage was statistically significant according to Kruskal-Wallis tests $(P<0.05)$ for all except fungal richness. there was strong congruence between the enrichment for $\mathrm{Bul}$ kholderiales in the rhizoplane and in rhizosphere soil in the conventional system. Differences in fungal rhizoplane communities between rhizoplanes in each system and between rhizoplane and bulk soil in each system were due primarily to the differential abundance of various Ascomycota and Basidiomycota OTUs (Supplementary Fig. S9 and S10).

We also conducted family-level analyses to assess differences in rhizoplane communities between the cropping systems to better assess patterns of enrichment. Vegetative stage 11 exhibited the greatest number of differentially abundant prokaryotic families between rhizoplanes in each system while R5 exhibited the fewest. At V11, families of the $\beta$-Proteobacteria (Burkholderiaceae, Oxalobacteraceae), $\alpha$-Proteobacteria (Sphingomonadacae, Bradyrhizobiacae, Rhizobiales), and Actinobacteria were commonly enriched in the conventional rhizoplane, whereas the Acidobacteria, Bacterioidetes, and Verrucomicrobia were commonly enriched in the diversified rhizoplane (Fig. 7A). For fungal rhizoplane communities, the most differentially abundant families occurred at stage V4, and at this stage the greatest number of differentially abundant families belonged to the Ascomycota, including unknown Hypocreales, Saccharomycetales, and Helotiales families (Fig. 7B).

\section{DISCUSSION}

In this study, we investigated the effects of a diversified (2-year rotation, manure amendments) and a conventional (2-year rotation, inorganic fertilization) cropping system on the dynamics of prokaryotic and fungal root-associated communities at four stages of maize development corresponding to varied degrees of $\mathrm{N}$ demand. The root-soil compartments examined here distinguished between the rhizosphere (soil at the root interface) and the rhizoplane (root surface), reflecting the continuum from the root surface outwards to the bulk soil, an important criterion defining root-associated microbial community research (Richter-Heitmann et al. 2016). Our results reveal that agricultural management (including differences in crop rotation, fertilization, tillage, and pesticide application) influences the assembly of prokaryotic and fungal communities near and on the root as the plant develops, with implications for our understanding of the rhizosphere effect and sustainable agricultural management.

Overall, root-associated community compositions, particularly within the rhizoplane, shifted significantly over the course of plant development, indicating root selection of microbial groups. These results are consistent with those of other studies showing that roots select for different microbial communities at different

TABLE 1

Permutational analysis of variance of $\beta$-diversity

\begin{tabular}{|c|c|c|c|c|}
\hline \multirow[b]{2}{*}{ Factor } & \multicolumn{2}{|c|}{ Prokaryotic } & \multicolumn{2}{|c|}{ Fungal } \\
\hline & $R^{2}$ & $P$ value & $R^{2}$ & $P$ value \\
\hline Cropping system & 0.065 & 0.0001 & 0.083 & 0.0001 \\
\hline Growth stage & 0.056 & 0.0001 & 0.095 & 0.0001 \\
\hline Cropping system $\times$ root proximity & 0.015 & 0.0011 & 0.018 & 0.0001 \\
\hline Root proximity $\times$ growth stage & 0.059 & 0.0001 & 0.066 & 0.0001 \\
\hline Cropping system $\times$ root proximity $\times$ growth stage & 0.014 & 0.5692 & 0.019 & 0.1789 \\
\hline
\end{tabular}


developmental stages (Chiarini et al. 1998; Gomes et al. 2003; Hannula et al. 2012; Houlden et al. 2008; Micallef et al. 2009; Xu et al. 2009). These shifts in community composition are likely caused by changes in root-exudation, which has been demonstrated for both prokaryotic and fungal communities in various plant species (Broeckling et al. 2008; Chaparro et al. 2014, 2013; Paterson et al. 2006). Our findings partially support our hypothesis that root-associated microbial communities would differ most between cropping systems at stages of rapid plant growth and high $\mathrm{N}$ demand. Prokaryotic rhizoplane communities in the two agricultural systems showed the least overlap in community composition and the most differentially abundant taxa at V11 when maize plants are known to be growing rapidly with high $\mathrm{N}$ uptake (Licht et al. 2011). These results provide evidence that, given the same plant genotype, agricultural management can have a marked impact on the trajectory of the prokaryotic rhizosphere community
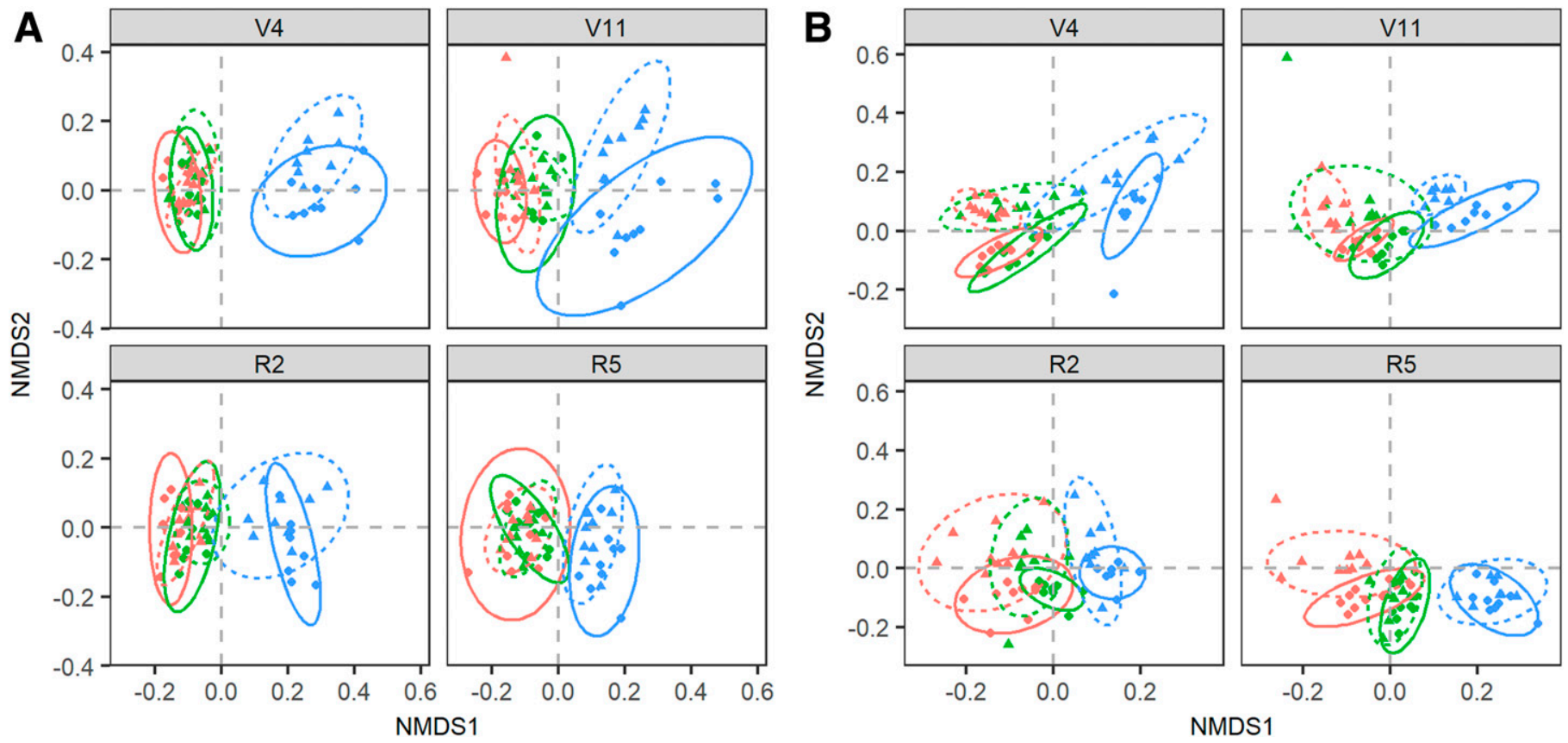

Fig. 3. Nonmetric multidimensional scaling (NMDS) ordinations of Bray-Curtis dissimilarities for microbial communities at the operational taxonomic unit level. A, Prokaryotic, stress $=0.142$, and B, fungal, stress $=0.201$. Ellipses indicate t-normal distributions of each treatment group. Conventional, $\bullet$ diversified, $\mathbf{\Delta}$; bulk soil, red; rhizosphere soil, green; and rhizoplane, blue.
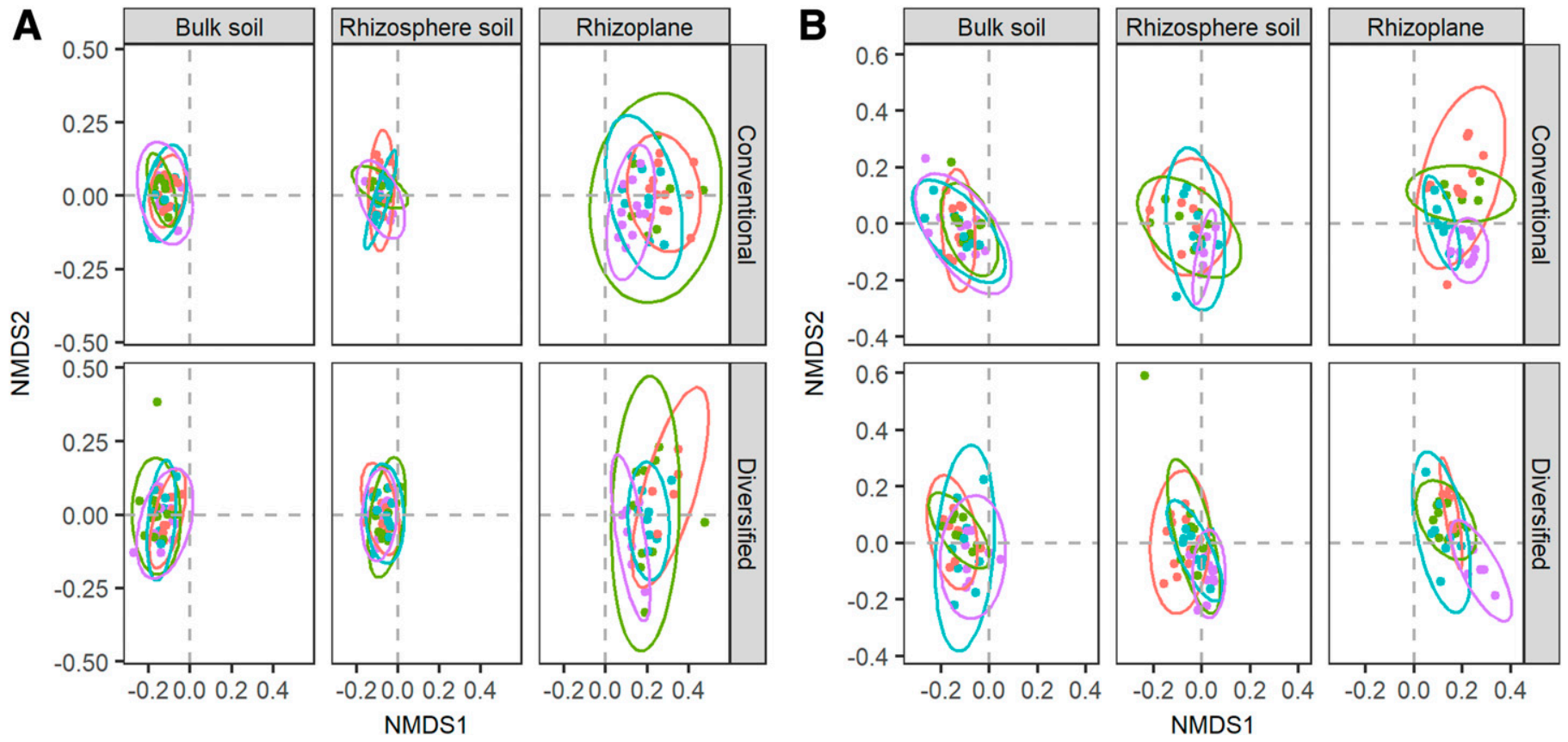

Fig. 4. Nonmetric multidimensional scaling (NMDS) ordinations of Bray-Curtis dissimilarities for bulk soil, rhizosphere soil, and rhizoplane microbial communities at each developmental stage. A, Prokaryotic, stress $=0.142$, and B, fungal, stress $=0.201$. Ellipses show the t-normal distributions of each treatment group. V4, red; V11, green; R2, blue; and R5, purple. 
structure as the plant grows. The convergence of rhizoplane communities between cropping systems at R2 and R5 suggests that the roots in the two systems select for similar communities at these stages, regardless of differences in the soil microbial seedbank. However, the types of root segments selected for sampling at R2 and R5 may have influenced these results as well; at those time points mature roots dominated the top layer of soil sampled and mature roots can shape root-associated prokaryotic communities differently than growing roots (DeAngelis et al. 2009). The microbiome of deeper roots or specific root types are likely to differ but were not studied in this experiment.

Families within the Verrucomicrobia and Acidobacteria were commonly and consistently more abundant in the diversified rhizoplane compared with the conventional rhizoplane at V11, when maize plants were at a stage of high nutrient demand and displayed the most differences between rhizoplane communities between the cropping systems. There is evidence from meta-analysis studies that these phyla have oligotrophic properties and are important for the breakdown of complex organic materials in undisturbed soils (Fierer et al. 2007, 2013). This is supported experimentally by a stable-isotope probing experiment that identified members of Verrucomicrobia as consumers of more complex C compounds, such as cellulose (Pepe-Ranney et al. 2016). Cultured members of these phyla have genomic pathways for the breakdown of complex, plant-derived polysaccharides (Martinez-Garcia et al. 2012; Ward et al. 2009). Additionally, members of the Acidobacteria and Verrucomicrobia are known to be capable of rhizosphere colonization (Nunes da Rocha et al. 2011, 2013; Tanaka et al. 2017). Considered together, their enrichment in the diversified system rhizoplane suggests that they may play an important role in decomposing complex organic material near the root. In juxtaposition to this, within the conventional system rhizoplane at stage V11, families of the Proteobacteria and Actinobacteria comprised most of the selectively enriched taxa. In stable-isotope probing experiments, many members of Proteobacteria and Actinobacteria were consistently identified as consumers of labile C substrates (Kramer et al. 2016; Pepe-Ranney et al. 2016). The widespread, selective increase in the abundance of Proteobacteria and Actinobacteria families in the conventional system rhizoplane compared with the diversified system rhizoplane may indicate a community more reliant on simple $\mathrm{C}$ substrates, less suited to complex $\mathrm{C}$ decomposition and, by extension, nutrient mineralization which is consistent with previous findings about the organic matter dynamics at this site (King and Hofmockel 2017; Osterholz et al. 2017). Management that relies exclusively on inorganic inputs may result in root selection of microbial communities more dependent on easily accessed $\mathrm{C}$ and disrupt the plant's ability to select for a prokaryotic community that mineralizes nutrients from existing organic matter.

Fungal root-associated communities of the two cropping systems differed most from one another at V4, when maize growth and $\mathrm{N}$ demand were not predicted to be high compared with other stages of plant development that we investigated (Licht et al. 2011). In this case, fungal communities did not appear to respond in concert with

TABLE 3

Contrasts of $\beta$-diversity between developmental stages ${ }^{z}$

\begin{tabular}{|c|c|c|c|c|c|c|}
\hline \multirow[b]{2}{*}{ Cropping system } & \multicolumn{2}{|c|}{ Bulk soil } & \multicolumn{2}{|c|}{$\begin{array}{l}\text { Rhizosphere } \\
\text { soil }\end{array}$} & \multicolumn{2}{|c|}{ Rhizoplane } \\
\hline & Conv. & Div. & Conv. & Div. & Conv. & Div. \\
\hline \multicolumn{7}{|l|}{ Prokaryotic } \\
\hline V4 versus V11 & 0.162 & 1.000 & 0.119 & 0.113 & 0.027 & 0.005 \\
\hline V11 versus $R 2$ & 0.826 & 1.000 & 0.497 & 0.956 & 0.022 & 0.027 \\
\hline $\mathrm{R} 2$ versus $\mathrm{R} 5$ & 1.000 & 1.000 & 1.000 & 1.000 & 0.016 & 0.027 \\
\hline \multicolumn{7}{|l|}{ Fungal } \\
\hline V4 versus V11 & 1.000 & 0.319 & 0.043 & 0.130 & 0.016 & 0.065 \\
\hline V11 versus $R 2$ & 0.329 & 1.000 & 0.027 & 0.027 & 0.081 & 0.027 \\
\hline $\mathrm{R} 2$ versus $\mathrm{R} 5$ & 1.000 & 1.000 & 0.259 & 0.027 & 0.005 & 0.043 \\
\hline
\end{tabular}

$z$ Values indicate $P$-adjusted values for each permutational analysis of variance test between consecutive time points. Conv. and Div. refer to conventional and diversified cropping systems, respectively.

TABLE 2

Contrasts of $\beta$-diversity between root-proximity levels ${ }^{z}$

\begin{tabular}{|c|c|c|c|c|c|c|c|c|}
\hline \multirow[b]{2}{*}{ Growth stage } & \multicolumn{2}{|c|}{ V4 } & \multicolumn{2}{|c|}{ V11 } & \multicolumn{2}{|c|}{$\mathrm{R} 2$} & \multicolumn{2}{|c|}{$\mathrm{R} 5$} \\
\hline & Conv. & Div. & Conv. & Div. & Conv. & Div. & Conv. & Div. \\
\hline \multicolumn{9}{|l|}{ Prokaryotic } \\
\hline$B$ versus RP & 0.027 & 0.005 & 0.027 & 0.027 & 0.022 & 0.027 & 0.027 & 0.027 \\
\hline RS versus $R P$ & 0.027 & 0.005 & 0.027 & 0.027 & 0.033 & 0.027 & 0.027 & 0.027 \\
\hline RS versus $R S$ & \multicolumn{2}{|c|}{0.027} & \multicolumn{2}{|c|}{0.027} & \multicolumn{2}{|c|}{0.049} & \multicolumn{2}{|c|}{0.027} \\
\hline RP versus $R P$ & \multicolumn{2}{|c|}{0.005} & \multicolumn{2}{|c|}{0.027} & \multicolumn{2}{|c|}{0.059} & \multicolumn{2}{|c|}{0.162} \\
\hline \multicolumn{9}{|l|}{ Fungal } \\
\hline$B$ versus $R S$ & 0.173 & 0.270 & 0.124 & 1.000 & 0.011 & 0.335 & 0.044 & 0.027 \\
\hline$B$ versus $R P$ & 0.005 & 0.022 & 0.038 & 0.043 & 0.032 & 0.027 & 0.027 & 0.043 \\
\hline RP versus RP & \multicolumn{2}{|c|}{0.032} & \multicolumn{2}{|c|}{0.113} & \multicolumn{2}{|c|}{0.070} & \multicolumn{2}{|c|}{0.043} \\
\hline
\end{tabular}

z Values indicate $P$-adjusted values for each permutational analysis of variance test between root proximity levels. Conv. and Div. refer to conventional and diversified, respectively. B, RS, and RP refer to bulk soil, rhizosphere soil, and rhizoplane, respectively. 
the shifting nutrient demands of the plant as did prokaryotic communities. At V4, when fungal rhizoplane communities were most distinct between cropping systems, most differentially abundant fungal taxa were families within the Ascomycota. This supports the findings of Gomes et al. (2003) who found that maize consistently selected for various groups within the Ascomycota at early stages of development. Ascomycota cover a broad range of ecological roles, including but not limited to saprotrophs, root endophytes, and plant pathogens (Schoch et al. 2009). It is possible that the fungal seedbank as shaped by agricultural management

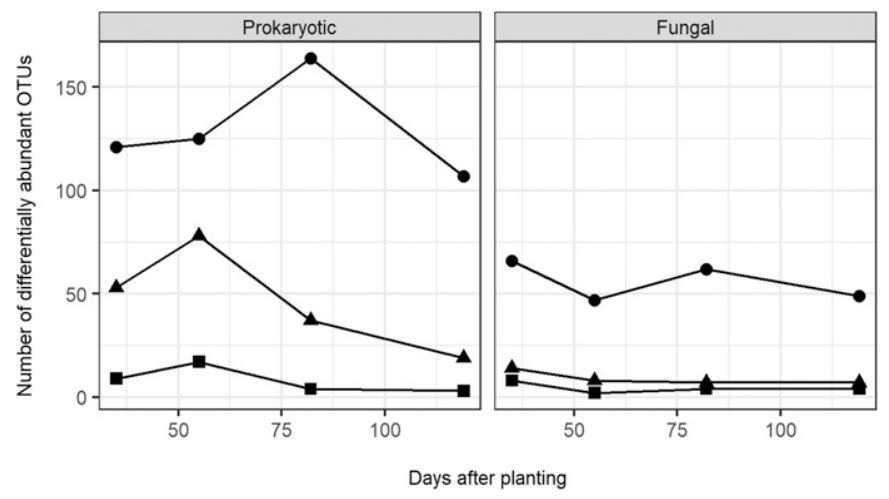

Fig. 5. Number of differentially abundant operational taxonomic units between the conventional and diversified cropping systems in the bulk soil $(\bullet)$, rhizosphere soil $(\boldsymbol{\square})$, and rhizoplane $(\boldsymbol{\Delta})$ after adjusting for underlying differences between bulk soil communities in each system. determines early recruitment of Ascomycota species by maize. Our finding that Ascomycota comprise the highest relative abundance and majority of differentially abundant taxa in the bulk soils of these two systems supports this possibility.

Root exudates diffuse from the root surface, influencing microbial communities up to $5 \mathrm{~mm}$ from the root under controlled conditions (Kandeler et al. 2002; Kuzyakov et al. 2003; Raynaud 2010). In both laboratory and field experiments, there are reports of distinct differences in community structure between rhizoplane or rhizosphere soil compared with bulk soil (Fernandez et al. 2016a, 6b; Kawasaki et al. 2016; Yin et al. 2017; Yurgel et al. 2017). We found that the rhizoplane microbial community differed most from the bulk soil microbiome, while differentiation between the rhizosphere and bulk soil microbiomes depended on plant developmental stage and agricultural management. This finding agrees with the theoretical work by Watt et al. (2006) who predicted that, under field conditions, the rhizoplane community would be much more responsive to root exudation than the bulk soil community due to its close proximity to the root. This highlights that, despite methodological challenges, studying the rhizosphere and rhizoplane separately can yield important insights into how roots, microbiota, and soil management interact that may help us leverage soil microbiomes to enhance $\mathrm{N}$ and other nutrient synchrony in agricultural systems.

Interestingly, in the conventional system, bulk and rhizosphere soil prokaryotic communities were significantly different from each other at only V11 and R2, suggesting there was a transient rhizosphere effect away from the root surface at these developmental stages in this system. The diversified system did not exhibit such

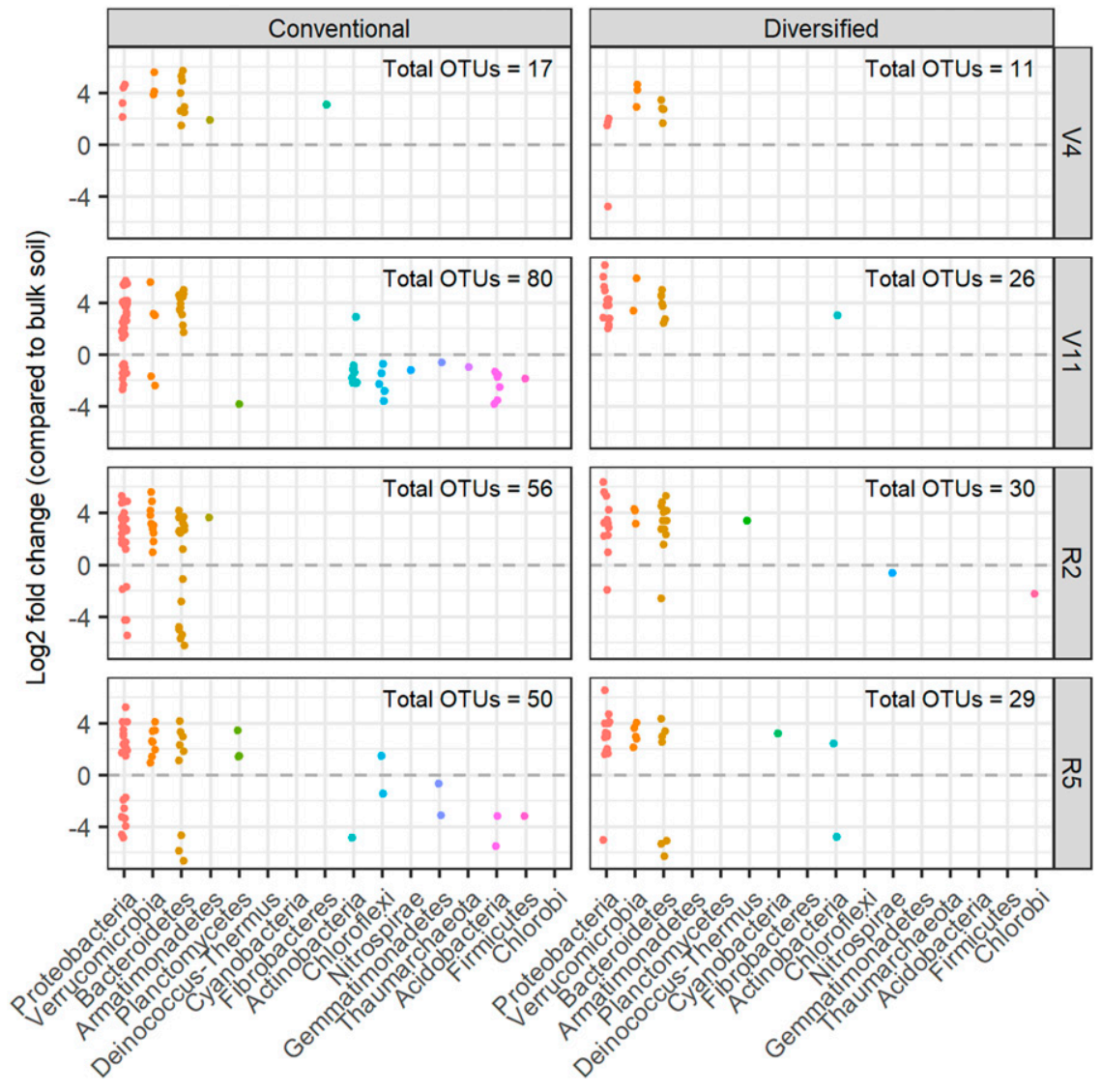

Fig. 6. Differentially abundant $(P$-adjusted $<0.01)$ prokaryotic operational taxonomic units between bulk and rhizosphere soils of conventional and diversified systems. Positive values indicated greater abundance in the rhizosphere compared with the bulk soil. 
differences at any stage. This could be a consequence of differential exudation by roots in the conventional system compared with the diversified system, analogous to how soil type and plant development influences the rhizosphere effect (Schreiter et al. 2014). We did not observe a similar effect on the rhizosphere fungal community, possibly because prokaryotes are more responsive to rhizodeposits than fungi or because fungi integrate over larger spatial scales due to their hyphal morphology. These findings suggest that the microbial community in the diversified system rhizosphere soil may be more similar to that of the bulk soil. This

A

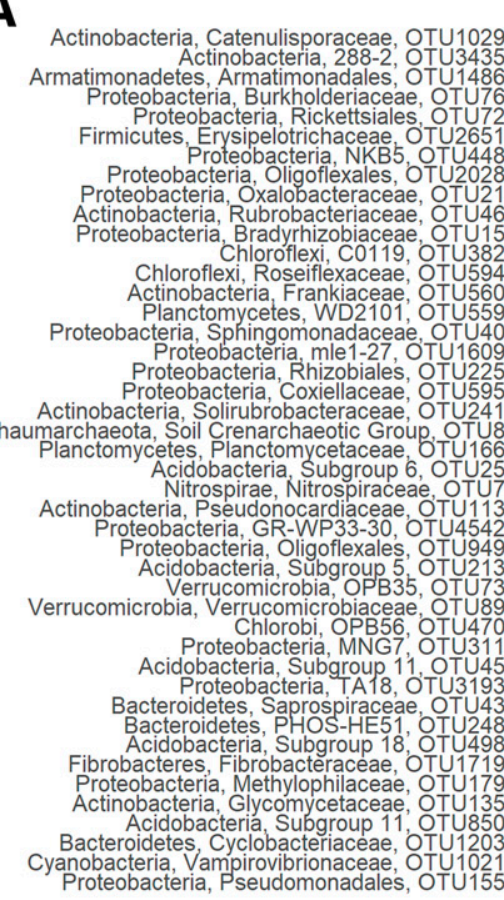

Actinobacteria, Catenulisporaceae, OTU1029imonadetes, Armatimonadales, OTU1486 roteobacteria, Burkholderiaceae, OTU76Orysipelotrichaceae, OTU2651-

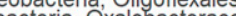

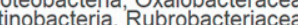
teobacteria. Bradyrhizobiacea ctinobacteria, Frankiaceae, Actinobacteria Solirubrobacteraceae, , Pseudonocardiaceae (1) Proteobacteria, MNG7, OTU311Acidobacteria, Subgroup 11, OTU45 Bacteroidetes, Saprospiraceae, OTU43Bacteroidetes, PHOS-HE51, OTU248Acidobacteria, Subgroup 18 robacteres, Fibrobacteraceae, Actinobacteria, Glycomycetaceae, inobacteria, Vampirovibrionaceae,

OTU1021.
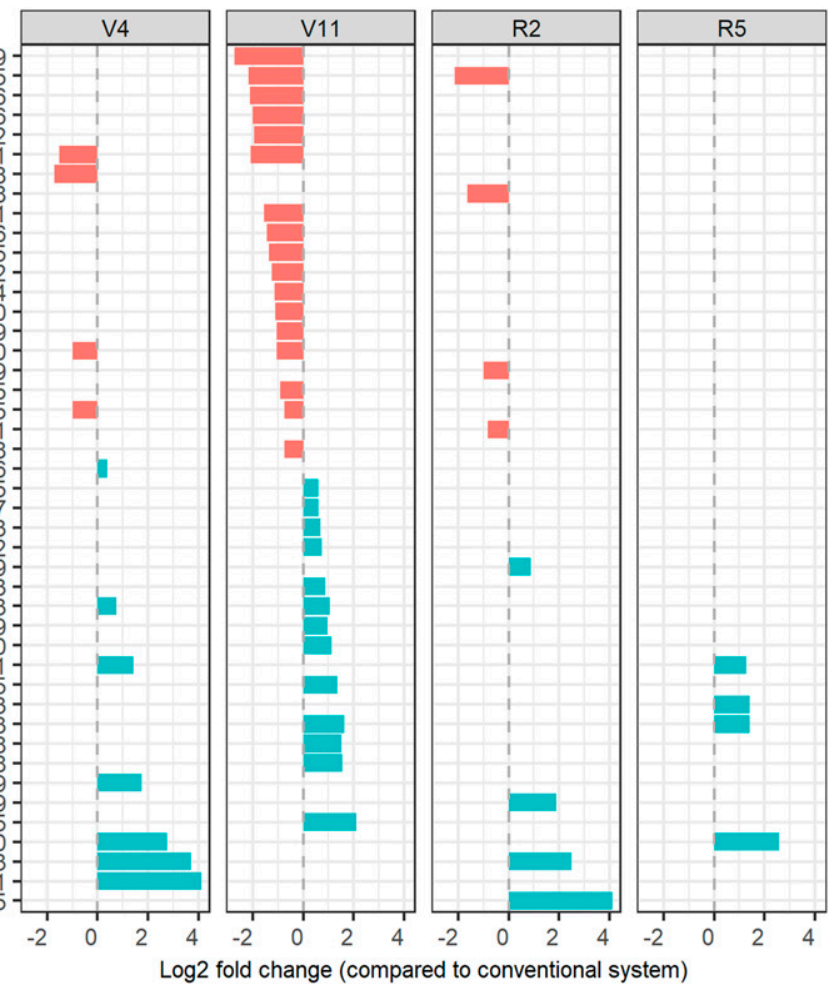

Log2 fold change (compared to conventional system)

B

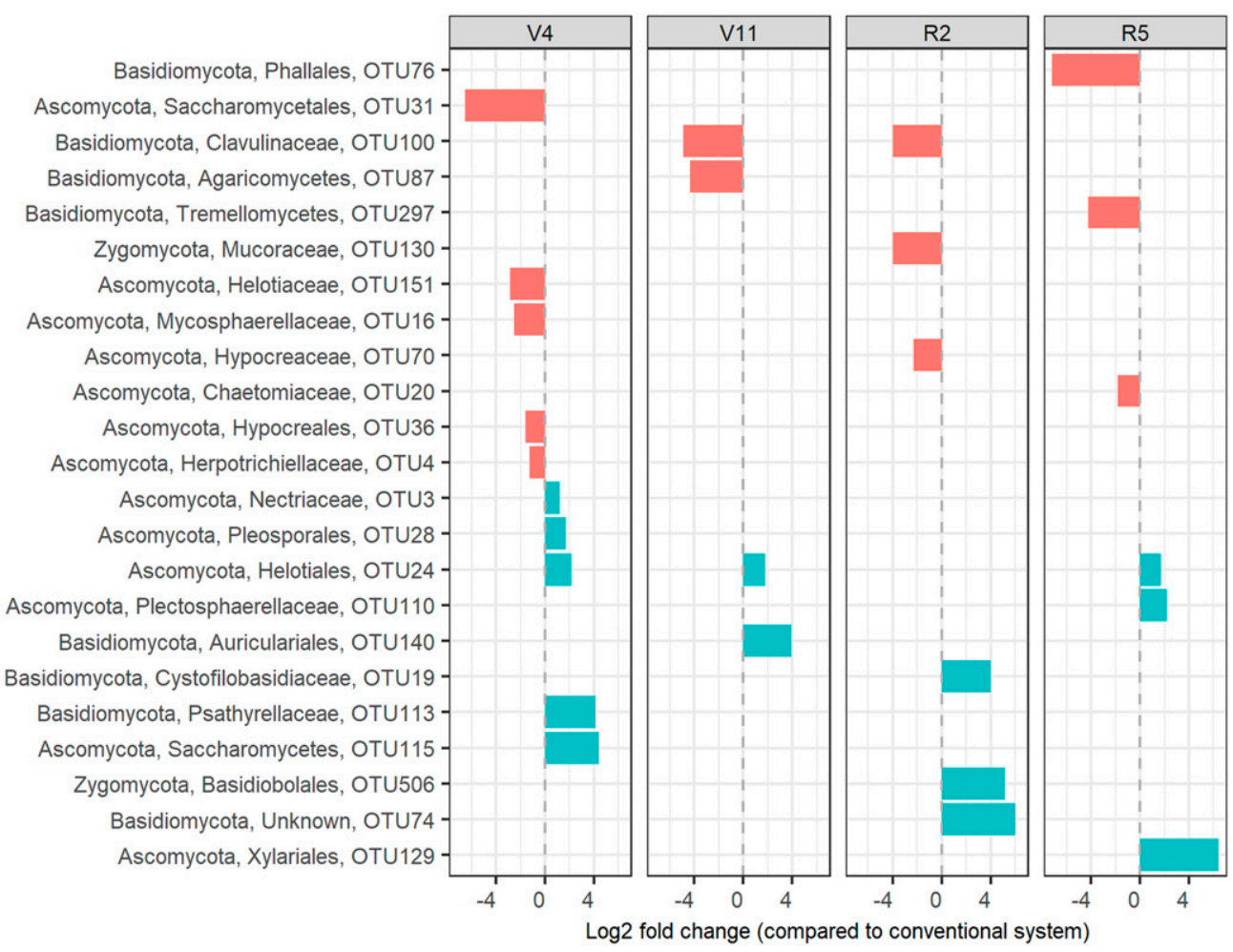

Fig. 7. Family-level differential abundance $(P$-adjusted $<0.01)$ between rhizoplane communities of differing cropping systems at each developmental stage after adjusting for underlying differences in bulk soil communities. A, Prokaryotic and B, fungal communities. The y-axis shows the phylum and family assignment of each taxa. If the family was unknown, the class or order assignment was substituted. A red bar indicates a lower abundance of the taxa in the diversified system, while a blue bar indicates a higher abundance of the taxa in the diversified system. 
may be evidence for the camouflage hypothesis, which proposed that microbial inoculant control of root disease could be a consequence of the rhizosphere community being more similar to the surrounding soil, masking the presence of the root to pathogens (Gilbert et al. 1994, 1993). This may contribute to the reduction in severity and incidence of soybean sudden death syndrome in the diversified system at the Marsden site (Leandro et al. 2018). The question remains whether the similarity between rhizosphere soil and bulk soil prokaryotic communities contributes to the crop yield benefit and reduced nutrient losses in the diversified system.

In conclusion, we showed that during a stage of fast growth with high nutrient demand, maize roots in differing cropping systems selected the most dissimilar prokaryotic communities on the rhizoplane. At this stage of development, in a manure-fed cropping system, the prokaryotic rhizoplane community selected for a higher abundance of members implicated in complex organic matter decomposition, while in a conventional, inorganically fed cropping system, the rhizoplane communities selected a higher abundance of taxa reliant on simple $\mathrm{C}$. This may be one mechanism by which the diversified system at the Marsden experimental site is able to support high productivity with reduced inorganic fertilization. In contrast, root-associated fungal communities appeared to be selected first based on the fungal seed bank supplied by the management regime and then converged to be highly similar regardless of agricultural management or predicted plant $\mathrm{N}$ demand, possibly due to strong selection by the maize roots. Surprisingly, prokaryotic rhizosphere communities did not differentiate from bulk soil communities consistently, while rhizoplane communities did, indicating that separate study of rhizoplanes and rhizospheres can yield important insights for agricultural management. For example, a transient rhizosphere effect away from the root surface occurred only within the conventional system, indicating differences in root selection between diversified and conventional systems that alter the rhizosphere effect.

\section{ACKNOWLEDGMENTS}

We thank G. Bay, G. Watson, A. Nease, B. Ritland, and C. Chen for their assistance in sample collection and processing; J. Brown for developing the Hundo pipeline and processing our sequencing data through it; and E. Bach's thoughtful discussion on multivariate statistical analysis.

\section{LITERATURE CITED}

Adesemoye, A. O., and Kloepper, J. W. 2009. Plant-microbes interactions in enhanced fertilizer-use efficiency. Appl. Microbiol. Biotechnol. 85: $1-12$.

Alexander, R. B., Smith, R. A., Schwarz, G. E., Boyer, E. W., Nolan, J. V., and Brakebill, J. W. 2008. Differences in phosphorus and nitrogen delivery to the Gulf of Mexico from the Mississippi River Basin. Environ. Sci. Technol. 42: 822-830.

Anders, S., and Huber, W. 2010. Differential expression analysis for sequence count data. Genome Biol. 11:R106.

Bakker, M. G. 2018. A fungal mock community control for amplicon sequencing experiments. Mol. Ecol. Resour. 12:1-16.

Baligar, V. C., Fageria, N. K., and He, Z. L. 2001. Nutrient use efficiency in plants. Commun. Soil Sci. Plant Anal. 32:921-950.

Baudoin, E., Benizri, E., and Guckert, A. 2002. Impact of growth stage on the bacterial community structure along maize roots, as determined by metabolic and genetic fingerprinting. Appl. Soil Ecol. 19:135-145.

Beck, S. M., and Gilmour, C. M. 1983. Role of wheat root exudates in associative nitrogen fixation. Soil Biol. Biochem. 15:33-38.

Berendsen, R. L., Pieterse, C. M. J., and Bakker, P. A. H. M. 2012. The rhizosphere microbiome and plant health. Trends Plant Sci. 17: 478-486.
Broeckling, C. D., Broz, A. K., Bergelson, J., Manter, D. K., and Vivanco, J. M. 2008. Root exudates regulate soil fungal community composition and diversity. Appl. Environ. Microbiol. 74:738-744.

Brown, J., Zavoshy, N., Brislawn, C .J., and McCue, L. A. 2018. Hundo: A Snakemake workflow for microbial community sequence data. PeerJ Preprints 6:e27272v1.

Bushnell, B. 2014. BBMap: A fast, accurate, splice-aware aligner. https:// sourceforge.net/projects/bbmap/

Camacho, C., Coulouris, G., Avagyan, V., Ma, N., Papadopoulos, J., Bealer, K., and Madden, T. L. 2009. BLAST+: architecture and applications. BMC Bioinformatics 10:421.

Carpenter, S. R., Caraco, N. F., Correll, D. L., Howarth, R. W., Sharpley, A. N., and Smith, V. H. 1998. Nonpoint pollution of surface waters with phosphorus and nitrogen. Ecol. Appl. 8:559-568.

Chaparro, J. M., Badri, D. V., Bakker, M. G., Sugiyama, A., Manter, D. K., and Vivanco, J. M. 2013. Root exudation of phytochemicals in Arabidopsis follows specific patterns that are developmentally programmed and correlate with soil microbial functions. PLoS One 8:e55731.

Chaparro, J. M., Badri, D. V., and Vivanco, J. M. 2014. Rhizosphere microbiome assemblage is affected by plant development. ISME 8: 790-803.

Chiarini, L., Bevivino, A., Dalmastri, C., Nacamulli, C., and Tabacchioni, S. 1998. Influence of plant development, cultivar and soil type on microbial colonization of maize roots. Appl. Soil Ecol. 8:11-18.

Davis, A. S., Hill, J. D., Chase, C. A., Johanns, A. M., and Liebman, M. 2012. Increasing cropping system diversity balances productivity, profitability and environmental health. PLoS One 7:e47149.

DeAngelis, K. M., Brodie, E. L., DeSantis, T. Z., Andersen, G. L., Lindow, S. E., and Firestone, M. K. 2009. Selective progressive response of soil microbial community to wild oat roots. ISME 3:168-178.

Edgar, R. C. 2010. Search and clustering orders of magnitude faster than BLAST. Bioinformatics 26:2460-2461.

Edgar, R. C. 2013. UPARSE: Highly accurate OTU sequences from microbial amplicon reads. Nat. Methods 10:996-998.

Fernandez, A. L., Shaeffer, C. C., Wyse, D. L., Staley, C., Gould, T. J., and Sadowsky, M. J. 2016b. Structure of bacterial communities in soil following cover crop and organic fertilizer incorporation. Appl. Microbiol. Biotechnol. 100:9331-9341.

Fernandez, A. L., Sheaffer, C. C., Wyse, D. L., Staley, C., Gould, T. J., and Sadowsky, M. J. 2016a. Associations between soil bacterial community structure and nutrient cycling functions in long-term organic farm soils following cover crop and organic fertilizer amendment. Sci. Total Environ. 566-567:949-959.

Fierer, N., Bradford, M. A., and Jackson, R. B. 2007. Toward an ecological classification of soil bacteria. Ecology 88:1354-1364.

Fierer, N., Ladau, J., Clemente, J. C., Leff, J. W., Owens, S. M., Pollard, K. S., Knight, R., Gilbert, J. A., and McCulley, R. L. 2013. Reconstructing the microbial diversity and function of pre-agricultural tallgrass prairie soils in the United States. Science 342:621-624.

Food and Agriculture Organization (FAO). 2017. World fertilizer trends and outlook to 2020. Summary Report. FAO, Rome, Italy.

Gdanetz, K., and Trail, F. 2017. The wheat microbiome under four management strategies, and potential for endophytes in disease protection. Phytobiomes J. $1: 158-168$.

Gilbert, G. S., Handelsman, J., and Parke, J. L. 1994. Root camouflage and disease control. Phytopathology 84:222-225.

Gilbert, G. S., Parke, J. L., Clayton, M. K., and Handelsman, J. 1993. Effects of an introduced bacterium on bacterial communities on roots. Ecology 74: 840-854.

Gomes, N. C. M., Fagbola, O., Costa, R., Rumjanek, N. G., Buchner, A., Mendona-Hagler, L., and Smalla, K. 2003. Dynamics of fungal communities in bulk and maize rhizosphere soil in the tropics. Appl. Environ. Microbiol. 69:3758-3766.

Hannula, S. E., de Boer, W., van Veen, J., Berg, G., and Young, J. 2012. A 3-year study reveals that plant growth stage, season and field site affect soil fungal communities while cultivar and GM-trait have minor effects. PLoS One 7:e33819.

Hargreaves, S. K., Williams, R. J., and Hofmockel, K. S. 2015. Environmental filtering of microbial communities in agricultural soil shifts with crop growth. PLoS One 10:1-14.

Hartman, K., van der Heijden, M. G. A., Wittwer, R. A., Banerjee, S., Walser, J.-C., and Schlaeppi, K. 2018. Cropping practices manipulate abundance patterns of root and soil microbiome members paving the way to smart farming. Microbiome 6:1-14 
Houlden, A., Timms-Wilson, T. M., Day, M. J., and Bailey, M. J. 2008. Influence of plant developmental stage on microbial community structure and activity in the rhizosphere of three field crops. FEMS Microbiol. Ecol. 65: 193-201.

Kandeler, E., Marschner, P., Tscherko, D., Singh Gahoonia, T., and Nielsen, N. E. 2002. Microbial community composition and functional diversity in the rhizosphere of maize. Plant Soil 238:301-312.

Kawasaki, A., Donn, S., Ryan, P. R., Mathesius, U., Devilla, R., Jones, A., and Watt, M. 2016. Microbiome and exudates of the root and rhizosphere of Brachypodium distachyon, a model for wheat. PLoS One 11:e0164533.

King, A. E., and Hofmockel, K. S. 2017. Diversified cropping systems support greater microbial cycling and retention of carbon and nitrogen. Agric. Ecosyst. Environ. 240:66-76.

Kõljalg, U., Nilsson, R. H., Abarenkov, K., Tedersoo, L., Taylor, A. F. S., Bahram, M., Bates, S. T., Bruns, T. D., Bengtsson-Palme, J., Callaghan, T. M., Douglas, B., Drenkhan, T., Eberhardt, U., Dueñas, M., Grebenc, T., Griffith, G. W., Hartmann, M., Kirk, P. M., Kohout, P., Larsson, E., Lindahl, B. D., Lücking, R., Martín, M. P., Matheny, P. B., Nguyen, N. H., Niskanen, T., Oja, J., Peay, K. G., Peintner, U., Peterson, M., Põldmaa, K., Saag, L., Saar, I., Schüßler, A., Scott, J. A., Senés, C., Smith, M. E., Suija, A., Taylor, D. L., Telleria, M. T., Weiss, M., and Larsson, K. H. 2013. Towards a unified paradigm for sequence-based identification of fungi. Mol. Ecol. 22: 5271-5277.

Kraiser, T., Gras, D. E., Gutiérrez, A. G., González, B., and Gutiérrez, R. A. 2011. A holistic view of nitrogen acquisition in plants. J. Exp. Bot. 62: 1455-1466.

Kramer, S., Dibbern, D., Moll, J., Huenninghaus, M., Koller, R., Krueger, D., Marhan, S., Urich, T., Wubet, T., Bonkowski, M., Buscot, F., Lueders, T., and Kandeler, E. 2016. Resource partitioning between bacteria, fungi, and protists in the detritusphere of an agricultural soil. Front. Microbiol. 7:1524.

Kuzyakov, Y., Raskatov, A., and Kaupenjohann, M. 2003. Turnover and distribution of root exudates of Zea mays. Plant Soil 254:317-327.

Kuzyakov, Y., and Xu, X. 2013. Competition between roots and microorganisms for nitrogen: Mechanisms and ecological relevance. New Phytol. 198: 656-669.

Leandro, L. F. S., Eggenberger, S., Chen, C., Williams, J., Beattie, G. A., and Liebman, M. 2018. Cropping system diversification reduces severity and incidence of soybean sudden death syndrome caused by Fusarium virguliforme. Plant Dis. 102:1748-1758.

Licht, M., Abendroth, L. J., Elmore, R. W., Boyer, M. J., and Marlay, S. K. 2011. Corn growth and development. PMR 1009, Iowa State University Extension, Ames, IA

Liebman, M., Gibson, L. R., Sundberg, D. N., Heggenstaller, A. H., Westerman, P. R., Chase, C. A., Hartzler, R. G., Menalled, F. D., Davis, A. S., and Dixon, P. M. 2008. Agronomic and economic performance characteristics of conventional and low-external-input cropping systems in the central corn belt. Agron. J. 100:600-610.

Love, M. I., Huber, W., and Anders, S. 2014. Moderated estimation of fold change and dispersion for RNA-seq data with DESeq2. Genome Biol. 15:550.

Martinez-Garcia, M., Brazel, D. M., Swan, B. K., Arnosti, C., Chain, P. S. G., Reitenga, K. G., Xie, G., Poulton, N. J., Gomez, M. L., Masland, D. E. D., Thompson, B., Bellows, W. K., Ziervogel, K., Lo, C. C., Ahmed, S., Gleasner, C. D., Detter, C. J., and Stepanauskas, R. 2012. Capturing single cell genomes of active polysaccharide degraders: An unexpected contribution of Verrucomicrobia. PLoS One 7:e35314.

McMurdie, P. J., and Holmes, S. 2013. phyloseq: An R package for reproducible interactive analysis and graphics of microbiome census data. PLoS One 8: e61217.

Meena, V. S., Meena, S. K., Vermad, J. P., Kumare, A., Aeronf, A., Mishraa, P. K., Bishta, J. K., Pattanayaka, A., Naveedg, M., and Dotaniya, M. L. 2017. Plant beneficial rhizospheric microorganism (PBRM) strategies to improve nutrients use efficiency: A review. Ecol. Eng. 107:8-32.

Micallef, S. A., Channer, S., Shiaris, M. P., and Colón-Carmona, A. 2009. Plant age and genotype impact the progression of bacterial community succession in the Arabidopsis rhizosphere. Plant Signal. Behav. 4:777-780.

Niu, S., Classen, A. T., Dukes, J. S., Kardol, P., Liu, L., Luo, Y., Rustad, L., Sun, J., Tang, J., Templer, P. H., Thomas, R. Q., Tian, D., Vicca, S., Wang, Y.-P., Xia, J., and Zaehle, S. 2016. Global patterns and substrate-based mechanisms of the terrestrial nitrogen cycle. Ecol. Lett. 19:697-709.

Nunes da Rocha, U., Plugge, C. M., George, I., van Elsas, J. D., van Overbeek, L. S., and Galantini, J. 2013. The rhizosphere selects for particular groups of Acidobacteria and Verrucomicrobia. PLoS One 8: e82443.
Nunes da Rocha, U., van Elsas, J. D., and van Overbeek, L. S. 2011. Verrucomicrobia subdivision 1 strains display a difference in the colonization of the leek (Allium porrum) rhizosphere. FEMS Microbiol. Ecol. 78: 297-305.

Oksanen, J., Blanchet, F.G., Friendly, M., Kindt, R., Legendre, P., Mcglinn, D., Minchin, P.R., O’Hara, R.B., Simpson, G.L., Solymos, P., Henry, M., Stevens, H., Szoecs, E., and Wagner, H., 2017. vegan: Community Ecology Package. R Package Version 2.4-3.

Osterholz, W. R., Liebman, M., and Castellano, M. J. 2018. Can soil nitrogen dynamics explain the yield benefit of crop diversification? Field Crops Res. 219:33-42.

Osterholz, W. R., Rinot, O., Liebman, M., and Castellano, M. J. 2017. Can mineralization of soil organic nitrogen meet maize nitrogen demand? Plant Soil 415:73-84.

Paterson, E., Gebbing, T., Abel, C., Sim, A., and Telfer, G. 2006. Rhizodeposition shapes rhizosphere microbial community structure in organic soil. New Phytol. 173:600-610.

Paulson, J. N., Stine, O. C., Bravo, H. C., and Pop, M. 2013. Differential abundance analysis for microbial marker-gene surveys. Nat. Methods 10:1200-1202.

Pepe-Ranney, C., Campbell, A. N., Koechli, C. N., Berthrong, S., and Buckley, D. H. 2016. Unearthing the ecology of soil microorganisms using a high resolution DNA-SIP approach to explore cellulose and xylose metabolism in soil. Front. Microbiol. 7:703.

Pfeiffer, S., Mitter, B., Oswald, A., Schloter-Hai, B., Schloter, M., Declerck, S., and Sessitsch, A. 2017. Rhizosphere microbiomes of potato cultivated in the High Andes show stable and dynamic core microbiomes with different responses to plant development. FEMS Microbiol. Ecol. 93: fiw 242 .

Quast, C., Pruesse, E., Yilmaz, P., Gerken, J., Schweer, T., Yarza, P., Peplies, J., and Glöckner, F. O. 2013. The SILVA ribosomal RNA gene database project: Improved data processing and web-based tools. Nucleic Acids Res. 41: D590-D596.

R Core Team. 2017. R: A Language and Environment for Statistical Computing.

Raynaud, X. 2010. Soil properties are key determinants for the development of exudate gradients in a rhizosphere simulation model. Soil Biol. Biochem. 42: 210-219.

Richardson, A. E., Barea, J. M., McNeill, A. M., and Prigent-Combaret, C. 2009. Acquisition of phosphorus and nitrogen in the rhizosphere and plant growth promotion by microorganisms. Plant Soil 321:305-339.

Richter-Heitmann, T., Eickhorst, T., Knauth, S., Friedrich, M. W., and Schmidt, H. 2016. Evaluation of strategies to separate root-associated microbial communities: A crucial choice in rhizobiome research. Front. Microbiol. 7:733.

Roberts, D. W. 2016. labdsv: Ordination and multivariate analysis for ecology. https://cran.r-project.org/web/packages/labdsv/index.html

Schimel, J. P., and Bennett, J. 2004. Nitrogen mineralization: Challenges of a changing paradigm. Ecology 85:591-602.

Schoch, C. L., Sung, G. H., López-Giráldez, F., Townsend, J. P., Miadlikowska, J., Hofstetter, V., Robbertse, B., Matheny, P. B., Kauff, F., Wang, Z., Gueidan, C., Andrie, R. M., Trippe, K., Ciufetti, L. M., Wynns, A., Fraker, E., Hodkinson, B. P., Bonito, G., Groenewald, J. Z., Arzanlou, M., Sybren de Hoog, G., Crous, P. W., Hewitt, D., Pfister, D. H., Peterson, K., Gryzenhout, M., Wingfield, M. J., Aptroot, A., Suh, S. O., Blackwell, M., Hillis, D. M., Griffith, G. W., Castlebury, L. A., Rossman, A. Y., Lumbsch, H. T., Lücking, R., Büdel, B., Rauhut, A., Diederich, P., Ertz, D., Geiser, D. M., Hosaka, K., Inderbitzin, P., Kohlmeyer, J., Volkmann-Kohlmeyer, B., Mostert, L., O’Donnell, K., Sipman, H., Rogers, J. D., Shoemaker, R. A., Sugiyama, J., Summerbell, R. C., Untereiner, W., Johnston, P. R., Stenroos, S., Zuccaro, A., Dyer, P. S., Crittenden, P. D., Cole, M. S., Hansen, K., Trappe, J. M., Yahr, R., Lutzoni, F., and Spatafora, J. W. 2009. The Ascomycota tree of life: A phylum-wide phylogeny clarifies the origin and evolution of fundamental reproductive and ecological traits. Syst. Biol. 58:224-239.

Schreiter, S., Ding, G. C., Heuer, H., Neumann, G., Sandmann, M., Grosch, R., Kropf, S., and Smalla, K. 2014. Effect of the soil type on the microbiome in the rhizosphere of field-grown lettuce. Front. Microbiol. 5:144.

Smil, V. 1999. Nitrogen in crop production: An account of global flows. Global Biogeochem. Cycles 13:647-662.

Smith, D. P., and Peay, K. G. 2014. Sequence depth, not PCR replication, improves ecological inference from next generation DNA sequencing. PLoS One 9:e90234.

Tanaka, Y., Matsuzawa, H., Tamaki, H., Tagawa, M., Toyama, T., Kamagata, Y., and Mori, K. 2017. Isolation of novel bacteria including rarely cultivated phyla, Acidobacteria and Verrucomicrobia, from the roots of emergent plants by simple culturing method. Microbes Environ. 32:288-292. 
Tomer, M. D., and Liebman, M. 2014. Nutrients in soil water under three rotational cropping systems, Iowa, USA. Agric. Ecosyst. Environ. 186:105-114.

Vitousek, P. M., Aber, J. D., Howarth, R. W., Likens, G. E., Matson, P. A., and Schindler, D. W. 1997. Human alteration of the global nitrogen cycle: Sources and consequences. Ecol. Appl. 7:737-750.

Ward, N. L., Challacombe, J. F., Janssen, P. H., Henrissat, B., Coutinho, P. M., Wu, M., Xie, G., Haft, D. H., Sait, M., Badger, J., Barabote, R. D., Bradley, B., Brettin, T. S., Brinkac, L. M., Bruce, D., Creasy, T., Daugherty, S. C., Davidsen, T. M., DeBoy, R. T., Detter, J. C., Dodson, R. J., Durkin, A. S., Ganapathy, A., Gwinn-Giglio, M., Han, C. S., Khouri, H., Kiss, H., Kothari, S. P., Madupu, R., Nelson, K. E., Nelson, W. C., Paulsen, I., Penn, K., Ren, Q., Rosovitz, M. J., Selengut, J. D., Shrivastava, S., Sullivan, S. A., Tapia, R., Thompson, L. S., Watkins, K. L., Yang, Q., Yu, C., Zafar, N., Zhou, L., and Kuske, C. R. 2009. Three genomes from the phylum Acidobacteria provide insight into the lifestyles of these microorganisms in soils. Appl. Environ. Microbiol. 75:2046-2056.

Watt, M., Silk, W. K., and Passioura, J. B. 2006. Rates of root and organism growth, soil conditions, and temporal and spatial development of the rhizosphere. Ann. Bot. 97:839-855
Wieland, G., Neumann, R., and Backhaus, H. 2001. Variation of microbial communities in soil, rhizosphere, and rhizoplane in response to crop species, soil type, and crop development. Appl. Environ. Microbiol. 67:5849-5854.

Xu, Y., Wang, G., Jin, J., Liw, J., Xhang, Q., and Liu, X. 2009. Bacterial communities in soybean rhizosphere in response to soil type, soybean genotype, and their growth stage. Soil Biol. Biochem. 41:919-925.

Yin, C., Mueth, N., Hulbert, S., Schlatter, D., Paulitz, T. C., Schroeder, K., Prescott, A., and Dhingra, A. 2017. Bacterial communities on wheat grown under long-term conventional tillage and no-till in the Pacific Northwest of the United States. Phytobiomes J. 1:83-90.

Yurgel, S. N., Douglas, G. M., Comeau, A. M., Mammoliti, M., Dusault, A., Percival, D., and Langille, M. G. I. 2017. Variation in bacterial and eukaryotic communities associated with natural and managed wild blueberry habitats. Phytobiomes J. 1:102-113.

Zhu, S., Vivanco, J. M., and Manter, D. K. 2016. Nitrogen fertilizer rate affects root exudation, the rhizosphere microbiome and nitrogen-use-efficiency of maize. Appl. Soil Ecol. 107:324-333. 